Journal of Systems Science and Information

Aug., 2016, Vol. 4, No. 4, pp. 343-353

DOI: $10.21078 /$ JSSI-2016-343-11

\title{
Empirical Analysis of AH-Shares
}

\author{
Hongxing YAO \\ School of Finance and Economics, Jiangsu University, Zhenjiang 212013, China; Faculty of Science, \\ Jiangsu University, Zhenjiang 212013, China \\ E-mail: hxyao@ujs.edu.cn \\ Kejuan ZHOU \\ Faculty of Science, Jiangsu University, Zhenjiang 212013, China \\ E-mail:703766719@qq.com
}

\begin{abstract}
Recent studies of correlations in Chinese stock market have mainly focused on the static correlations in financial time series, and then we pay great attention to investigate their dynamic evolution of correlations. Our paper reports on topology of $41 \mathrm{AH}$-shares companies traded on Shanghai and Hong Kong Stock Exchange in Chinese stock market. We apply the concept of minimum spanning tree (MST) and hierarchical tree (HT) to analyze and reveal the dynamic evolution of correlations between different market sectors for the period 2008-2014. From these trees, we can detect that significantly industry clustering effects are in the stock network. We measure the linkage of different companies geared to different industrial sectors. We observe the evolution of AH-shares companies in the stock network based on the moving window technique and investigate the correlations by calculating the correlation coefficient distribution, mean correlation coefficient and mean distance of these companies with time. Therefore, through our analysis, we find that companies working in the same branch of production tend to make up cluster. The results present the difference and similarity between different industry sectors in different time periods.
\end{abstract}

Keywords complex network; dynamic evolution; minimum spanning tree; hierarchical tree

\section{Introduction}

Recently, the complex network theory is widely used to investigate the stock market. A number of works ${ }^{[1-3]}$ have been done to analyze the topological properties of stock networks and the correlations among stocks based on those methods including Random Matrix Theory $(\mathrm{RMT})^{[4-7]}$, Principal Component Analysis $(\mathrm{PCA})^{[8]}$, Planar Maximally Filtered Graph $(\mathrm{PMFG})^{[9]}$, correlation based on clustering ${ }^{[10]}$, and hierarchical structure ${ }^{[11]}$. For example, Mantegna ${ }^{[12,13]}$ studied the hierarchical structure in financial markets based on minimum spanning tree and hierarchical tree with the time series of stock returns. Utsugi ${ }^{[14]}$ investigated correlated groups of issues in these markets and proposed a refined method to identify correlated groups based on RMT. Tolga ${ }^{[15]}$ reported on complexity of major UK companies between 2006 and 2010 by using Hierarchical structure method approach. Numerous researches at home and

Received August 8, 2015, accepted January 7, 2016

Supported by the National Natural Science Foundation of China (71271107, 71271103) 
abroad have focused on global stock markets. Du, et al. ${ }^{[16]}$ found the comovement effect of stock market by the community structure and obtained the accurate block in the Chinese stock market. Zhang, et al. ${ }^{[17]}$ applied Partial Correlation Coefficients and Simple Correlation Coefficients to construct network of stocks. Hu, et al. ${ }^{[18]}$ discussed the dynamic characteristics of the stock networks evolution based on calculating the correlation coefficient, setting threshold and combining the financial index system. Gui, et al. ${ }^{[19]}$ detected some characters of dynamic communities in each time window based on Blondel's algorithm. Yang, et al. ${ }^{[20]}$ analyzed the linkage effects among industry sectors in Chinese stock market before and after the financial crisis. Onnela, et al. ${ }^{[21]}$ investigated the dynamics of market correlations and taxonomy analysis in detail. Michael ${ }^{[11]}$ has shown that hierarchical structure methods can be used to analyze the topology of foreign exchange markets and found that the price determination structure of international currency markets is tree like and sparsely clustered. This implies dynamic behavior related to complex networks can be applied to currency markets. In other words, the stock market is a complex network because of their price fluctuations. Therefore, in this research, we analyze the dynamic evolution of AH-shares companies in Chinese stock market by virtue of graphs. A-shares purchased and traded on the Shanghai and Shenzhen stock exchanges are shares of Renminbi currency, while H-shares listed in Hong Kong are foreign capital stocks. We choose those shares published both in Chinese Mainland and Hong Kong to represent Chinese stock market. In this paper we also need to take account of Chinese industry characteristic and circumstances to explore the topological characteristics of Chinese stock market. We can find financial business companies, industry companies, service industry companies and energy industry companies have cluster behavior. In the case of the emerging market, there are only a few large companies separated from other companies of the market. In this work, we also find individual stocks are clustered based on their individual sectors to which they belong.

In Section 2, we describe the sample datasets. In Section 3, we show the methodology used in this paper. In Sections 4 and 5, we present the dynamic behaviors and our empirical results and analysis of this study. Finally, conclusions are given in Section 6.

\section{Sample Dataset}

In this paper, to better understand the correlation structures under different market environments, we select $41 \mathrm{AH}$-shares stocks traded on Shanghai and Hong Kong Stock Exchange from March 10, 2008 to November 24, 2014 (excluding weekends and market closed dates). The dataset is filtered as follows: 1) Make sure that the stocks have enough number of trading days; 2) Delete those stocks from the dataset when the stock has missing closing prices for more than 10 continuous trading days; 3) Use the closing prices of the previous trading day to fill those missing closing prices. According to the above principles, 41 stocks remain in the sample dataset and each stock has 1632 observations. Stocks and codes are listed in Table 1. In accordance with the China Securities Index Co. Ltd, the listed companies are classified into 7 industries by the China Industry Classification Standard (CICS). These companies are divided into 12 Financial and Real Estate Industry, 14 Industry and Service Industry, 3 Public Service Industry, 6 Energy Industry, 2 Consumer Staple Industry and 4 Material Industry.

The database analyzed in this paper contains the daily stock data of $41 \mathrm{AH}$-shares companies 
in China traded on both Shanghai Stock Exchange (SHSE) and Hong Kong Exchanges and Clearing Limited (HKEx). The data source is from NetEase Finance.

Table 1 Code of listed companies

\begin{tabular}{|c|c|c|}
\hline Company name & A /H shares code & Code \\
\hline Huaneng Power Intl & $600011 / 0902$ & HPI \\
\hline Anhui Expressway & $600012 / 0995$ & $\mathrm{AE}$ \\
\hline China Minsheng Bank & $600016 / 1988$ & CMSB \\
\hline China Shipping Development & $600026 / 1138$ & CSDC \\
\hline Huadian Power & $600027 / 1071$ & $\mathrm{HP}$ \\
\hline SINOPEC & $600028 / 0386$ & SINOPEC \\
\hline China Southern Airlines & $600029 / 1055$ & $\mathrm{CZ}$ \\
\hline Citic Securities & $600030 / 6030$ & CITICS \\
\hline China Merchants Bank & $600036 / 3968$ & $\mathrm{CMB}$ \\
\hline Yanzhou Coal Mining Company & $600188 / 1171$ & $\mathrm{YZC}$ \\
\hline Shanghai Fosun Pharmaceutical & $600196 / 2196$ & SFP \\
\hline Jiangxi Copper & $600362 / 0358$ & JCCL \\
\hline Jiangsu Expressway Company & $600377 / 0177$ & $\mathrm{JE}$ \\
\hline Shenzhen Expressway & $600548 / 0548$ & $\mathrm{SE}$ \\
\hline Anhui Conch Cement & $600585 / 0914$ & $\mathrm{ACC}$ \\
\hline Tsingtao Beer & $600600 / 0168$ & TB \\
\hline Kunming Machine & $600806 / 0300$ & KM \\
\hline Magang stocks trading Co. & $600808 / 0323$ & MS \\
\hline Haitong Securities & $600837 / 6837$ & HS \\
\hline TCEPY & $600874 / 1065$ & TCEPY \\
\hline Dongfang Electric & $600875 / 1072$ & DEC \\
\hline China Shenhua Energy & $601088 / 1088$ & CSE \\
\hline Air China & $601111 / 0753$ & $\mathrm{CA}$ \\
\hline China Railway Construction & $601186 / 1186$ & CRCC \\
\hline Bank of Communications & $601328 / 3328$ & BOCOM \\
\hline Guangzhou-Shenzhen Railway & $601333 / 0525$ & GSH \\
\hline China Railway Engineering Corporation & $601390 / 0390$ & CRE \\
\hline Industrial and Commercial Bank of China & $601398 / 1398$ & $\mathrm{ICBC}$ \\
\hline Beijing North Star & $601588 / 0588$ & BEIJF \\
\hline Chinese aluminum industry & $601600 / 2600$ & CHALCO \\
\hline China Pacific Insurance & $601601 / 2601$ & $\mathrm{CP}$ \\
\hline China Life Insurance & $601628 / 2628$ & LFC \\
\hline China Oilfield Services & $601808 / 2883$ & COSL \\
\hline PetroChina & $601857 / 0857$ & CNPC \\
\hline China Shipping Container Lines & $601866 / 2866$ & CSCL \\
\hline ChinaCoal & $601898 / 1898$ & $\mathrm{CC}$ \\
\hline China Cosco Holdings & $601919 / 1919$ & COSCO \\
\hline China Construction Bank & $601939 / 0939$ & $\mathrm{CCB}$ \\
\hline Bank of China & $601988 / 3988$ & $\mathrm{BOC}$ \\
\hline Datang Intl Power & $601991 / 0991$ & DIP \\
\hline China Citic Bank & $601998 / 0998$ & CITICB \\
\hline
\end{tabular}




\section{Methods}

\subsection{Calculation Method}

Before we quantify the cross-correlations among stocks, we use logarithmic yields to calculate correlation coefficients and distances among stocks. Here, we take logarithm of the prices because the fluctuation of stock prices is typically given by the geometric Brownian motion. The logarithmic yield for a given stock $i$ is defined as

$$
S_{i}(t)=\ln p_{i}(t)-\ln p_{i}(t-1)
$$

where $p_{i}(t)$ is the closing price of stock $i$ at time $t, p_{i}(t-1)$ is the closing price of stock $i$ at time $t-1$, and $t$ is in units of one day. Then the correlation coefficient between two stock logarithmic yields $S_{i}(t)$ and $S_{j}(t)$ is defined as

$$
C_{i j}=\frac{\left\langle S_{i}(t) S_{j}(t)\right\rangle-\left\langle S_{i}(t)\right\rangle\left\langle S_{j}(t)\right\rangle}{\sqrt{\left(\left\langle S_{i}^{2}(t)\right\rangle-\left\langle S_{i}(t)\right\rangle^{2}\right)\left(\left\langle S_{j}^{2}(t)\right\rangle-\left\langle S_{j}(t)\right\rangle^{2}\right)}}
$$

where $\left\langle S_{i}(t)\right\rangle$ is the average return of stock $i$ in period $n,\left\langle S_{i}(t)\right\rangle=\frac{1}{n} \sum_{i=1}^{n} S_{i}(t) ;\left\langle S_{i}(t) S_{j}(t)\right\rangle$ stands for the average yield of their product at the same time. The correlation coefficient is used to measure the dependence between the return series in the whole period of the sample stocks. In this paper, there are $N=41$ sample stocks. Accordingly, we obtain a correlation matrix $C$ with $41 \times 41$ correlation coefficients as elements. Every element $C_{i j}$ corresponds to the correlation between stock $i$ and stock $j$. By the definition of $C_{i j}$, every element is restricted to the domain $-1 \leq C_{i j} \leq 1$; if $-1 \leq C_{i j} \leq 0$, then the stocks are negative correlated; if $0 \leq C_{i j} \leq 1$, then the stocks are correlated; and if $C_{i j}=0$, the stocks are uncorrelated. Mantegna ${ }^{[12]}$ introduced a definition of the similarity distance between stock $i$ and stock $j$ rigorously calculated by the $D$ matrix:

$$
D_{i j}=\sqrt{2\left(1-C_{i j}\right)}
$$

The correlation coefficient defined above is utilized to calculate the dependence between the return series in the whole period of the sample dataset. The distances $D_{i j}$ are used to create a complete undirected graph,where each node corresponds to a listed company, and each edge stands for the similarity distance between two companies. In addition, we use the moving window technique to further study the dynamic evolution of the stock correlations with time $t$. The size $T$ of the moving window is fixed to be 200 trading days, i.e., about one year. Equation (2) is applied to calculate the correlation coefficients over a subset of return series within the moving window $[t-T+1, t]$. The moving distance is step by step. For instance, in consideration of our sample dataset, which is from $3 / 10 / 2008$ to $11 / 24 / 2014$, the first moving window covers the period from $3 / 10 / 2008$ to $12 / 25 / 2008$. Hence, our dataset is divided into 1433 windows.

Then the mean correlation coefficient is expressed as follows:

$$
\left\langle C_{i j}\right\rangle=\frac{1}{N(N-1)} \sum_{i \neq j} C_{i j}
$$

The mean distance is expressed as follows:

$$
\left\langle D_{i j}\right\rangle=\frac{1}{N-1} \sum D_{i j}
$$


Numbers of hierarchical clustering methods used to detect the information associated to the correlation matrix can be found in the literature. It is worth noting that hierarchical clustering methods can as well be applied to distance matrices. Our analysis is a filtering procedure based on the estimation of the subdominant ultrametric distance associated with a metric distance obtained from the correlation coefficient matrix of a set of $n$ stocks. The subdominant ultrametric ${ }^{[22]}$ is the ultrametric structure closest to the original metric structure. With the above definition $D_{i j}$ fulfills the three axiom of a metric 1) $D_{i j} \geq 0, D_{i j}=0$ if and only if $i=j$; 2) $D_{i j}=D_{j i}$ (symmetry); 3) $D_{i j} \leq D_{i k}+D_{k j}$. In mathematics, ultrametric distances $D_{i j}$ are distances satisfying the inequality $D_{i j} \leq \max \left\{D_{i k}, D_{j k}\right\}$. And then the subdominant ultrametric matrix can be obtained from generating its minimum spanning tree and associating with the metric space. We can use Matlab7.0 software to obtain the hierarchical tree by using the equation written above.

\subsection{Hierarchical Tree and Minimum Spanning Tree}

Using the minimum spanning tree (MST) and hierarchical tree (HT), we study the structure and dynamics of the listed companies and explore the hierarchical structure in various time series.

The distance matrix $D_{i j}$ is then used to determine the MST connecting the $n$ stocks. The MST is a graph without loops connecting all the $n$ nodes with the shortest $n-1$ links among all the links. The MST is also a graph of a set of elements in the node arrangement in a given metric space, e.g., an ultrametric space.

Kruskal algorithm is a greedy algorithm. A brief description of the Kruskal algorithm is as follow: View each node as an isolated branch and sort all the possible edges in the order of increasing weights. Kruskal's minimum spanning tree algorithm starts with an empty graph and then attempts to add edges in increasing order of weight. Repeatedly add the next lightest edge that doesn't produce a loop until $n-1$ edges are added by the time the minimum spanning tree is formed.

\section{Dynamical Behavior}

From above, we first analyze the distribution of the elements $C_{i j}$ of the correlation matrix to obtain the statistical properties. In Figure1, the probability density function $P\left(c_{i j}\right)$ of the correlation coefficients calculated from the return series of $41 \mathrm{AH}$-shares stocks evolved with the time $t$ is shown. It will be found in the figure that the center of the distribution clearly deviates from zero. We observe the values of the coefficient $C_{i j}$ located at the peaks of $P\left(c_{i j}\right)$ are significantly positive. As $t$ tends to 2009 and 2011, the peaks of $P\left(c_{i j}\right)$ show two local maximum of $C_{i j}$. The valleys of $P\left(c_{i j}\right)$ are probably at 2010 and 2012. On September 15, 2008, the fifth-largest US investment bank Lehman Brothers declared bankruptcy and Merrill Lynch was also acquired by Bank of America, which caused the global financial crisis. Moreover, the stock markets around the world, certainly including the Chinese stock market, have been hit hard in the downturn. This indicates that stock price variations are more likely to be correlated around the market crashes. 


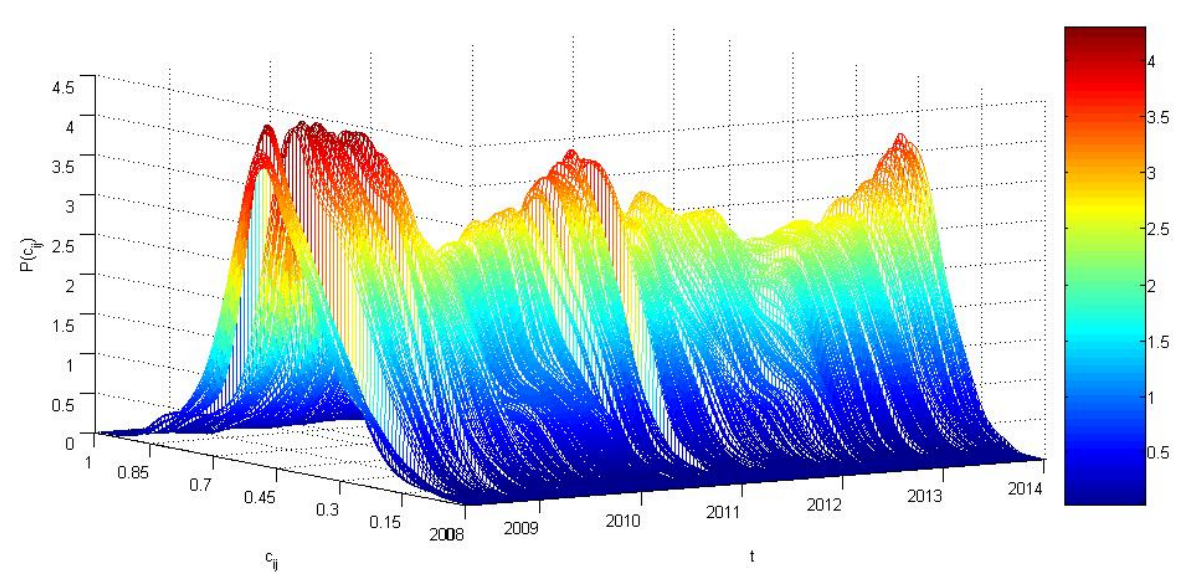

Figure 1 Dynamics of correlation coefficient distribution

To further verify the dependence of the stock correlations on the time and the dynamic stability of the network, we also compute the mean correlation coefficient $\left\langle C_{i j}\right\rangle$ and the mean distance $\left\langle D_{i j}\right\rangle$ in the moving window. In Figures 2, 3 we present a plot of the mean correlation coefficients and the mean distances among all stocks.

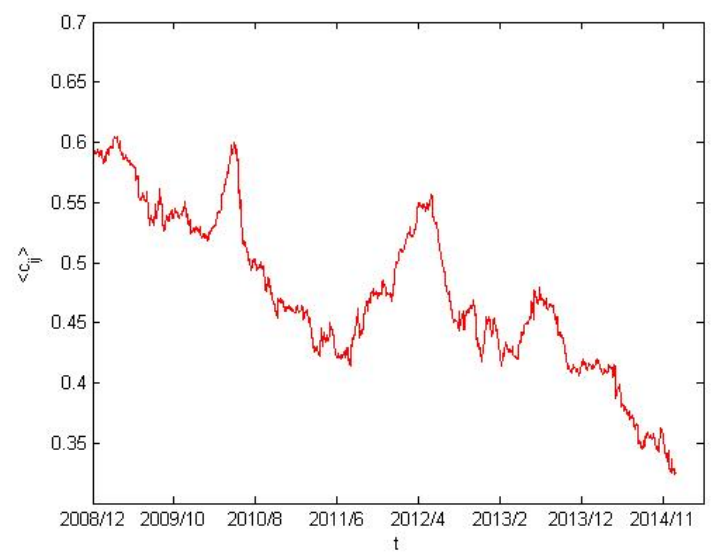

Figure 2 Dynamic of mean correlation coefficient of AH-shares companies

As shown in Figures 2 and 3, we can find that the mean correlation coefficients and the mean distances have a relatively strong negative correlation. With the reduction of distances, correlations among the stocks increased dramatically and the relevance became more closely. By observing the dynamic of mean correlation coefficient figure we can see that for the years 2008, 2010 and 2012 the mean value of the correlation coefficient matrix increased dramatically. In fact, during these years there was a sudden decline in the Chinese stock market (as in the rest of the world). This large increase of the mean correlation value suggests that during this crisis period all stocks were so highly correlated. Figure 2 shows that the mean correlation coefficients are stable in most of the time,but we can also see great volatility during three periods, which 
are late 2008, mid-2010 and early 2012. So in this paper we use March 10, 2010 and March 13, 2012 as dividing points. We can see clearly that the mean correlation coefficient is in decline as a whole, while the mean distance is gradually increasing over time. It is enough to suggest that the correlation of stock market is increasingly weaking.

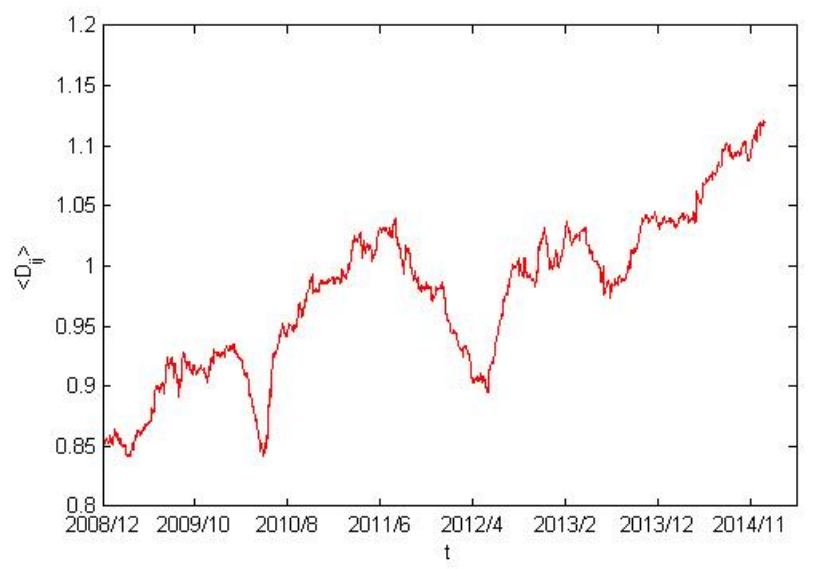

Figure 3 Dynamic of mean distances of AH-shares companies

\section{Empirical Results and Analysis}

In this section, using $41 \mathrm{AH}$-shares companies price indicators, we show the MSTs and their HTs, and investigate the topology and structure of the correlation networks in Chinese stock market. A number of researches about the engaged connections among the listed companies can be implemented by analyzing the MSTs and HTs. Analyzing these trees it can be checked that companies working in the same branch of production tend to cluster. The MSTs showed in Figures 4, 5 and 6 are obtained by using Kruskal's algorithm as we have discussed above. The HTs of the subdominant ultrametric space associated with the MST are shown in Figures 7, 8 and 9. Through a comprehensive comparative analysis of the $41 \mathrm{AH}$-shares company indices minimum spanning tree structures, we note that obvious clusters exist in all periods, especially among the similar industries. It will be found in the research that both MSTs and HTs show significantly different structures in three periods: From March 10, 2008 to March 10, 2010, from March 11, 2010 to March 12, 2012, and from March 13, 2012 to November 24, 2014. Notice that almost three groups are easily identified, financial and estate industry market (black dash line circle), industry and service industry market (green dash line circle), and energy industry market (blue dash circle). See Figure 4, inside each group, we find that financial and estate industry (black colour nodes) are linked to energy industry (blue colour nodes). Energy industry (blue colour nodes) are also linked to industry and service industry (green colour nodes). Thus the energy industry market acts as a bridge between the financial and estate industry market and the industry and service industry market. In Figure 4, the different colours represent the different sectors of economic activity. The black nodes mean the stocks included in Financial and Real Estate Industry. The blue nodes denote stocks from Energy Industry. The green nodes stand for Industry and Service Industry. Red nodes mean the stocks which are not included. 


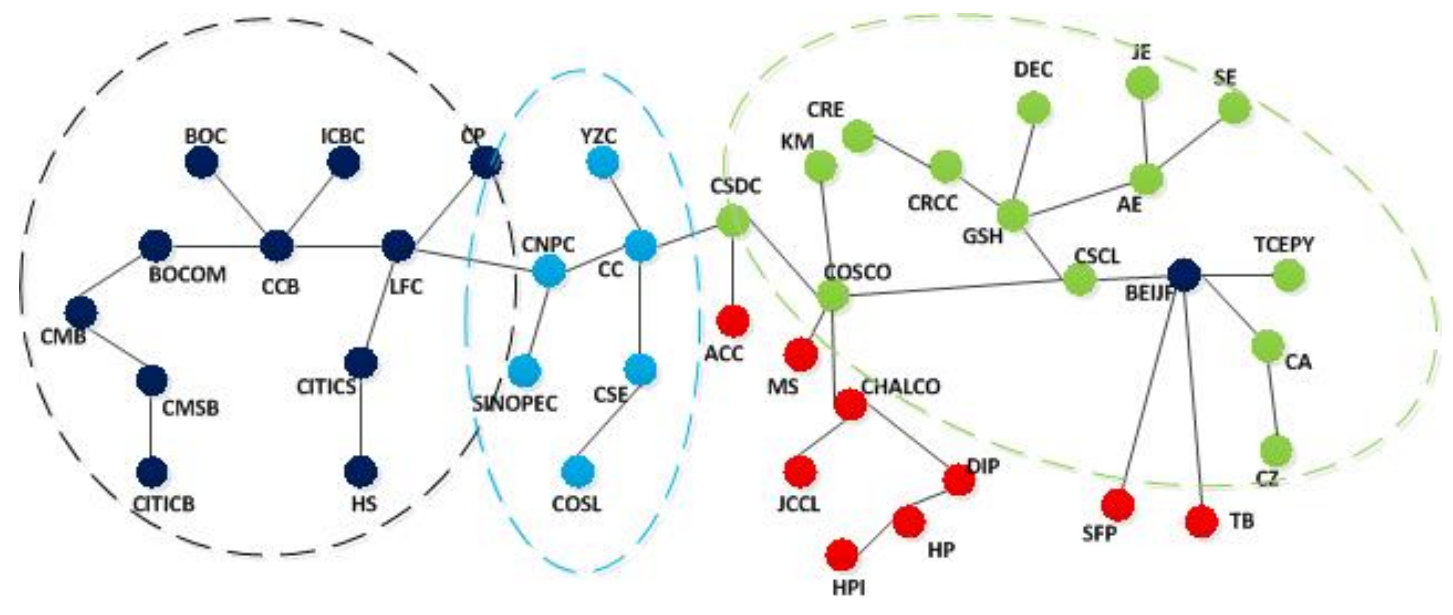

Figure 4 Minimum spanning tree of AH-Shares Companies from 2008/3/10 to 2010/3/10

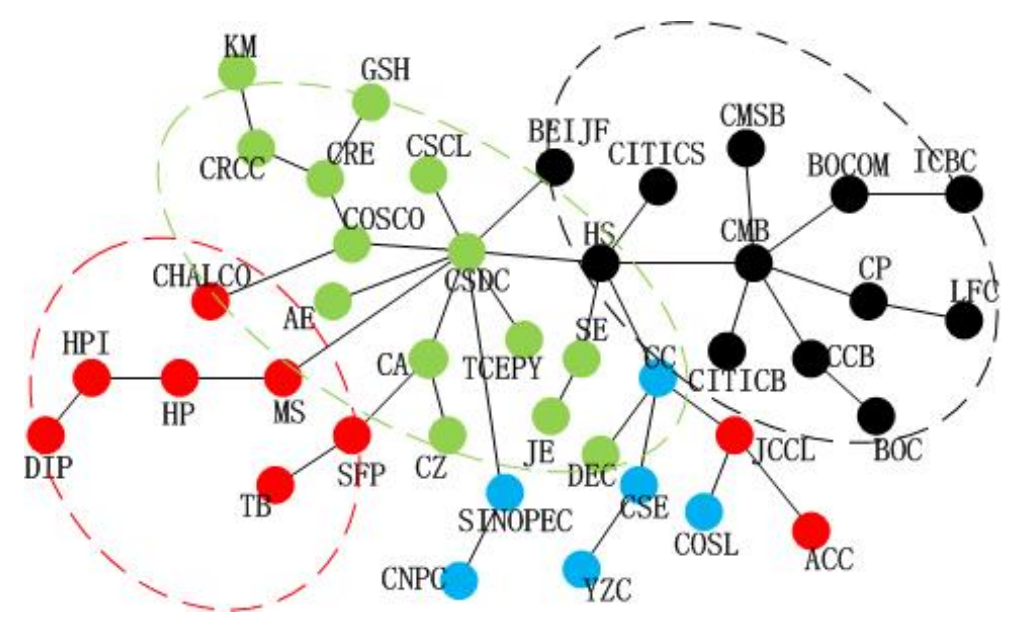

Figure 5 Minimum spanning tree of AH-Shares Companies from 2010/3/11 to 2012/3/12

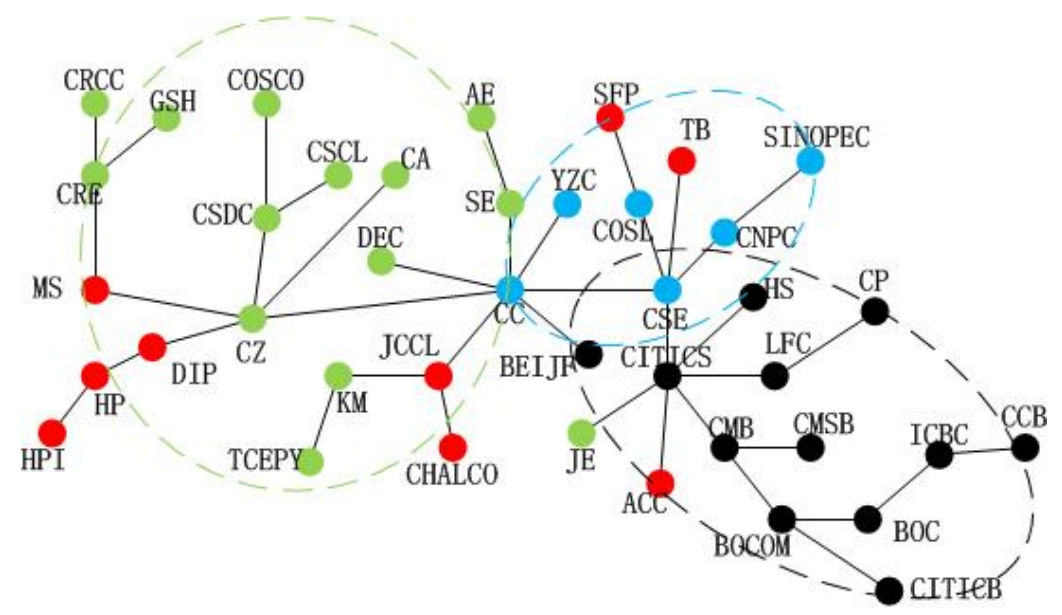

Figure 6 Minimum spanning tree of AH-Shares Companies from 2012/3/13 to 2014/11/24 


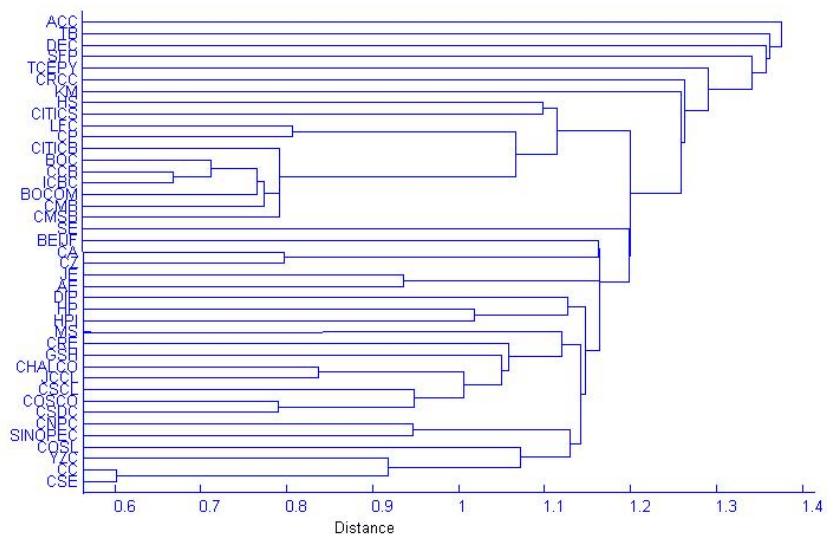

Figure 7 Hierarchical tree of AH-Shares Companies from 2008/3/10 to 2010/3/10

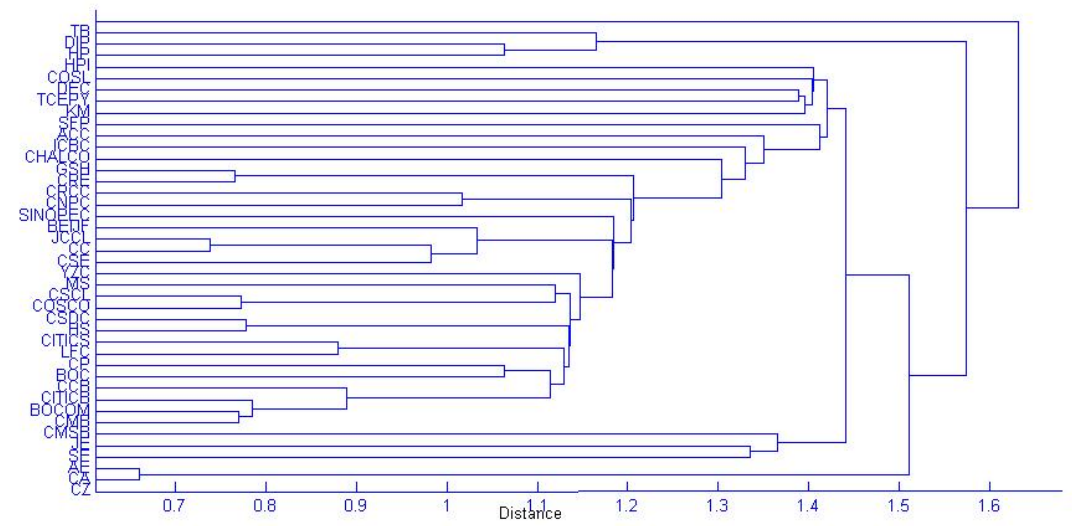

Figure 8 Hierarchical tree of AH-Shares Companies from 2010/3/11 to 2012/3/12

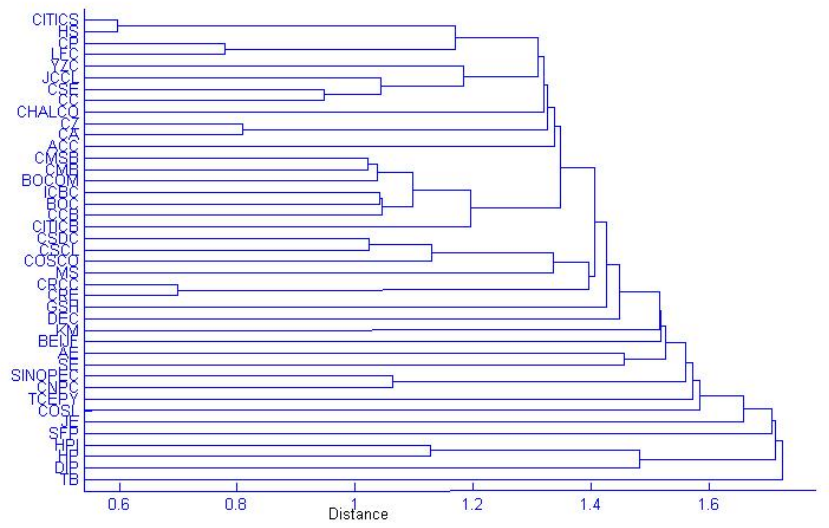

Figure 9 Hierarchical tree of AH-shares Companies from 2012/3/13 to 2014/11/24 
In order to find the main important companies appeared in different periods, we investigate the degrees of the core nodes in the networks. The degrees of the core nodes in different periods are shown in Table 2, 3 and 4. As Tables show, we can find that ChinaCoal (CC) is still a central node in different networks. Meanwhile, the degree of ChinaCoal rises from 4 to 9 , indicating that its linkage effect with other companies has improved. While it must be pointed out that Beijing North Star (BEIJF), China Shipping Development (CSDC) and ChinaCoal (CC) belong to financial and estate industry, industry and service industry and energy industry respectively. Thus it can be seen that the center of the market is quite different in different periods due to the various market environment. By comparing those MSTs, we find that Figure 4 shows a structure that differs greatly from that in Figure 5 especially the industry and service industry market (green dash line circle). Figure 6 also shows that the boundaries separating companies.

Table 2 Degrees of core nodes

\begin{tabular}{cccccc}
\hline \multicolumn{5}{c}{$2008.3 .10-2010.3 .10$} \\
\hline node & BEIJF & COSCO & CCB & LFC & CC \\
degree & 5 & 5 & 4 & 4 & 4 \\
\hline
\end{tabular}

Table 3 Degrees of core nodes 2010.3.11-2012.3.12

\begin{tabular}{ccccc}
\hline node & CSDC & CMB & HS & CC \\
degree & 9 & 6 & 5 & 4 \\
\hline
\end{tabular}

Table 4 Degrees of core nodes

\begin{tabular}{ccccc}
\hline \multicolumn{5}{c}{$2012.3 .13-2014.11 .24$} \\
\hline node & CC & CITICS & CZ & CSE \\
degree & 7 & 6 & 5 & 5 \\
\hline
\end{tabular}

In hierarchical tree, each horizontal line denotes a listed company, while the height of the vertical line represents the ultrametric distance at which the two companies are merged. The HTs can show the classification information of all these companies and present the linkage effects among company clusters. The hierarchical trees can reveal the correlation degrees of companies where the ones strongly linked in the network are at the same level. For instance, Air China (CA) and China Southern Airlines (CZ) are always at the same level in Figures 7-9, indicating that they are closely related. However, the distance between $\mathrm{CA}$ and $\mathrm{CZ}$ and other companies has gradually become larger, indicating that their linkage with other companies has weakened. Moreover, Tsingtao Beer (TB) is almost always at the highest level in all the three periods. It is enough to show that TB's linkage with other companies is rather weak. From Figure 7, we can see that the distance between ChinaCoal (CC) and China Shenhua Energy (CSE) is the smallest of the sample, indicating a strong relationship between these two companies. Financial and estate companies and industry companies are very close so that they make up the cluster.

\section{Conclusion}

In this paper, we investigated the clustering properties of individual industrial sectors in MST and HT structures estimated from a correlation matrix describing 41 individual AH- 
shares stocks listed on Shanghai and Hong Kong. The dynamic evolution study of stock network stability indicates that the network is stable in most of the time and the network has significantly fluctuation during the years 2008, 2010 and 2012, which this paper takes as dividing points. We find that the stock returns behave more collectively in volatile periods, showing the distribution of correlation coefficients centered around larger positive coefficients and larger values of mean correlation coefficient as the time approaches the three big crashes.

\section{References}

[1] Ricardo C, Claire G G, Brian L. The evolution of interdependence in world equity markets: Evidence from minimum spanning trees. Physica A, 2007, 376: 455-466.

[2] Antonios G, Panos A. Correlation study of the Athens Stock Exchange. Physica A, 2007, 380: 399-410.

[3] Jung W S, Chae S, Yang J, et al. Characteristics of the Korean stock market correlations. Physica A, 2006, 361: 263-271.

[4] Laloux L, Cizeau P, Bouchaud J P, et al. Noise dressing of financial correlation matrices. Physical Review Letters, 199, 83: 1467-1470.

[5] Plerou V, Gopikrishnan P, Rosenow B, et al, Stanley HE. Universal and nonuniversal properties of cross correlations in financial time series. Physical Review Letters, 1999, 83: 1471-1474.

[6] Eom C, Oh G, Jung W S, et al. Topological properties of stock networks based on minimal spanning tree and random matrix theory in financial time series. Physica A, 2009, 388: 900-906.

[7] Eom C, Kwon O, Jung W S, et al. The effect of a market factor on information flow between stocks using the minimal spanning tree. Physica A, 2010, 389: 1643-1652.

[8] Kritzman M, Li Y Z, Page S, et al. Principal components as a measure of systemic risk. Portfolio Management, 2011, 37: 112-126.

[9] Tumminello M, Aste T, Di Matteo T, et al. A tool for filtering information in complex systems. Proceedings of the National Acadamic Sciences, 2005, 102: 10421-10426.

[10] Huan C, Yong M, Sai-Ping L. Analysis of network clustering behavior of the Chinese stock market. Physica A, 2014, 414: 360-367.

[11] Michael J N, Lawrence C R, Brendan J M. Topology of foreign exchange markets using hierarchical structure methods. Physica A, 2007, 382: 199-208.

[12] Mantegna R N. Hierarchical structure in financial markets. European Physical Journal B, 1999, 11: $193-197$.

[13] Bonanno G, Caldarelli G, Lillo F, et al. Topology of correlation-based minimal spanning trees in real and model markets. Physical Review E, 2003, 68: 046130.

[14] Utsugi A, Ino K, Oshikawa M. Random matrix theory analysis of cross correlations in financial markets. Physical Review E, 2004, 70: 026110.

[15] Tolga U, Mustafa K, Ayoub S, et al. Complexity of major UK companies between 2006 and 2010: Hierarchical structure method approach. Physica A, 2012, 391: 5121-5131.

[16] Du C, Wang X, Qiu L. Block \& Comovement Effect of Stock Market in Financial Complex Network. Social Informatics and Telecommunications Engineering, 2009, 5: 1248-1260.

[17] Zhang J, Chen Y, Zhai D. Network analysis of Shanghai sector in Chinese stock market based on partial correlation. Information Management and Engineering, 2010: 321-324.

[18] Hu P, Qi J, Wang B. The characteristics analysis of the stock network based on weighted relative values: An example of information service industry. International Conference on Science and Social Research, 2013, 64: 411-416.

[19] Gui X, Li L, Cao J. Dynamic communities in stock market. Abstract and Applied Analysis, 2014: 723482.

[20] Yang R, Li X, Zhang T. Analysis of linkage effects among industry sectors in China's stock market before and after the financial crisis. Physica A, 2014, 411: 12-20.

[21] Onnela J P, Chakraborti A, Kaski K. Dynamics of market correlations: Taxonomy and portfolio analysis. Physical Review E, 2003, 68: 056110.

[22] Huang F X, Gu J, Li Y X, et al. Linkages and dynamic stability of the national of global primary stock index before and after the financial crisis. Systems Engineering — Theory \& Practice, 2010, 30(10): 1729-1740. 